BEDRATYUK L. ${ }^{1}$, LUNO N. $^{2}$

\title{
SOME PROPERTIES OF GENERALIZED HYPERGEOMETRIC APPELL POLYNOMIALS
}

Let $x^{(n)}$ denotes the Pochhammer symbol (rising factorial) defined by the formulas $x^{(0)}=1$ and $x^{(n)}=x(x+1)(x+2) \cdots(x+n-1)$ for $n \geq 1$. In this paper, we present a new real-valued Appelltype polynomial family $A_{n}^{(k)}(m, x), n, m \in \mathbb{N}_{0}, k \in \mathbb{N}$, every member of which is expressed by mean of the generalized hypergeometric function $F_{q}\left[\begin{array}{c}a_{1}, a_{2}, \ldots, a_{p} \\ b_{1}, b_{2}, \ldots, b_{q}\end{array} \mid z\right]=\sum_{k=0}^{\infty} \frac{a_{1}^{(k)} a_{2}^{(k)} \ldots a_{p}^{(k)}}{b_{1}^{(k)} b_{2}^{(k)} \ldots b_{q}^{(k)}} \frac{z^{k}}{k !}$ as follows

$$
A_{n}^{(k)}(m, x)=x_{k+p}^{n} F_{q}\left[\begin{array}{c}
a_{1}, a_{2}, \ldots, a_{p},-\frac{n}{k^{\prime}},-\frac{n-1}{k}, \ldots,-\frac{n-k+1}{k} \\
b_{1}, b_{2}, \ldots, b_{q}
\end{array} \mid \frac{m}{x^{k}}\right]
$$

and, at the same time, the polynomials from this family are Appell-type polynomials.

The generating exponential function of this type of polynomials is firstly discovered and the proof that they are of Appell-type ones is given. We present the differential operator formal power series representation as well as an explicit formula over the standard basis, and establish a new identity for the generalized hypergeometric function. Besides, we derive the addition, the multiplication and some other formulas for this polynomial family.

Key words and phrases: Appell sequence, Appell polynomial, generalized hypergeometric polynomial, generalized hypergeometric function.

${ }^{1}$ Khmelnytskyi National University, 11 Instytytska str., 29016, Khmelnytskyi, Ukraine

2 Vasyl' Stus Donetsk National University, 21 600-richya str., 21021, Vinnytsia, Ukraine

E-mail: leonid.uk@gmail.com (Bedratyuk L.), lunio@ukr.net (Luno N.)

\section{INTRODUCTION}

In [4], P. Appell presented polynomial sequence $\left\{A_{n}(x)\right\}, n=0,1,2, \ldots$, such that $\operatorname{deg} A_{n}(x)=n$ and satisfying the identity

$$
A_{n}^{\prime}(x)=n A_{n-1}(x)
$$

where $A_{0}(x) \neq 0$, which is called the Appell polynomial sequence.

An arbitrary Appell polynomial sequence possesses an exponential generating function

$$
A(t) e^{x t}=\sum_{n=0}^{\infty} A_{n}(x) \frac{t^{n}}{n !}
$$

here $A(t)$ is a formal power series

$$
A(t)=a_{0}+a_{1} t+a_{2} \frac{t^{2}}{2 !}+\cdots+a_{n} \frac{t^{n}}{n !}+\cdots, \quad a_{0} \neq 0 .
$$

$\mathrm{y} \Delta \mathrm{K} 519.4$

2010 Mathematics Subject Classification: 11B83, 33C45.

(C) Bedratyuk L., Luno N., 2020 
The Appell-type polynomials $A_{n}(x)$ are expressed in the terms of $\left\{a_{n}\right\}$ as follows

$$
A_{n}(x)=\sum_{i=0}^{n}\left(\begin{array}{l}
n \\
i
\end{array}\right) a_{n-i} x^{i}
$$

The simplest example of Appell-type polynomials is the monomial sequence $\left\{x^{n}\right\}, n=$ $0,1, \ldots$; other examples are the Bernoulli, the Euler polynomials and the Hermite polynomials. For more examples one can consult $[1,11]$.

The Appell-type polynomials perform a large variety of features and are widely spread at the different areas of mathematics, namely, at special functions, general algebra, combinatorics and number theory. Recently, the Appell-type polynomials are of big interest. The modern researches give the alternative definitions of Appell-type polynomials and apply new approaches based, for instance, on the determinant method or in Pascal matrix method (see, e.g., $[3,16])$. Consequently, many new properties of those polynomials are described and a great deal of identities involving Appell-type polynomials are obtained (see $[2,6,7])$.

Let us recall that the generalized hypergeometric function is defined as follows

$$
{ }_{p} F_{q}\left[\begin{array}{c}
a_{1}, a_{2}, \ldots, a_{p} \\
b_{1}, b_{2}, \ldots, b_{q}
\end{array} \mid z\right]=\sum_{k=0}^{\infty} \frac{a_{1}^{(k)} a_{2}^{(k)} \ldots a_{p}^{(k)}}{b_{1}^{(k)} b_{2}^{(k)} \ldots b_{q}^{(k)}} \frac{z^{k}}{k !}
$$

where $a_{1}, a_{2}, \ldots, a_{p}, b_{1}, b_{2}, \ldots, b_{p}$ are complex parameters and none of $b_{i}$ equals to a non-positive integer or zero, $x^{(n)}$ denotes the Pochhammer symbol (or rising factorial) defined by $x^{(n)}=x(x+1)(x+2) \cdots(x+n-1)$ for $n \geq 1$ and $x^{(0)}=1$. Further on, we denote the generalized hypergeometric function by $F_{q}$ for brevity.

We note that the Gauss hypergeometric function ${ }_{2} F_{1}$ and the Kummer hypergeometric function ${ }_{1} F_{1}$ are the partial cases of (2).

Apart from the Appell-type polynomials, there exist some polynomial families, which admit representation via the partial cases of the generalized hypergeometric function, i.e., the Jacobi polynomials ([1])

$$
P_{n}^{(\alpha, \beta)}(z)=\frac{(\alpha+1)^{(n)}}{n !}{ }_{2} F_{1}\left[\begin{array}{c|c}
-n, n+\alpha+\beta+1 & \frac{1-z}{2} \\
\alpha+1 & .
\end{array}\right.
$$

At the same time, there exists a number of the Appell-type polynomial families, which also admit the representation via partial cases of the Gauss hypergeometric function. It is known [1], that the Laguerre polynomials $L_{n}(x)$ are presented as follows

$$
L_{n}(x)={ }_{1} F_{1}\left[\begin{array}{c|c}
-n & x \\
1 &
\end{array}\right]
$$

Remarkably, the Hermite polynomials $H_{n}(x)$ are simply expressed in the terms of those functions ([8])

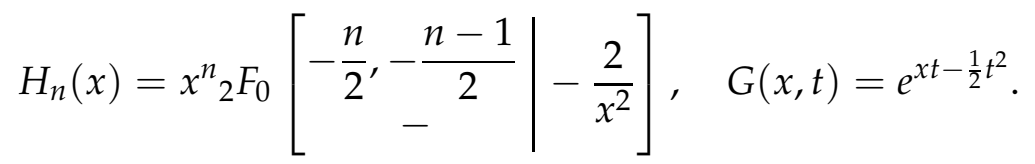

The natural way of generalisation of the Hermitte polinomials is to expand the array of ratios for another denominators, it was made in [10], the authors obtained the Gould-Hopper 
polynomials $g_{n}^{m}(x, h)$, with $G(x, t)=e^{x t+h t^{m}}$, which could be also expressed in the terms of the generalized hypergeometric function as follows

$$
g_{n}^{m}(x, h)=x_{m}^{n} F_{0}\left[\begin{array}{c}
\left.-\frac{n}{m},-\frac{n-1}{m}, \ldots,-\frac{n-m+1}{m} \mid \frac{(-1)^{m} h m^{m}}{x^{m}}\right] . \\
-
\end{array}\right]
$$

The aim of this paper is to find a polynomial family, which would be the Appell-type one and admit the generalized hypergeometric function representation simultaneously. Still, there exist the polynomial families, which have the needed representation, e.g., the generalized hypergeometric polynomials $f_{n}\left(a_{i} ; b_{j} ; x\right)$, studied at [9], such that

$$
f_{n}\left(a_{i} ; b_{j} ; x\right)={ }_{p+2} F_{q+2}\left[\begin{array}{c}
-n, n+1, a_{1}, a_{2}, \ldots, a_{p} \\
1, \frac{1}{2}, b_{1}, b_{2}, \ldots, b_{q}
\end{array} \mid x^{n}\right], \quad n \in \mathbb{N}_{0}
$$

and the incomplete hypergeometric polynomials associated with generalized incomplete hypergeometric function, studied at [13], but they both are not the Appel-type polynomials.

The difference between all mentioned classes of polynomials, depending, if they are of Appell-type or not and if they possess the generalized hypergeometric function representation or do not, has motivated the title of the paper.

Therefore, let us give the following

Definition 1. Let $\Delta(k,-n)$ denotes the array of $k$ ratios $-\frac{n}{k},-\frac{n-1}{k}, \ldots,-\frac{n-k+1}{k}, n \in \mathbb{N}_{0}$, $k \in \mathbb{N}$. Then we call the polynomial family

$$
A_{n}^{(k)}(m, x)=x_{k+p}^{n} F_{q}\left[\begin{array}{c|c}
a_{1}, a_{2}, \ldots, a_{p}, \Delta(k,-n) \\
b_{1}, b_{2}, \ldots, b_{q}
\end{array} \mid \frac{m}{x^{k}}\right], \quad n, m \in \mathbb{N}_{0}, \quad k \in \mathbb{N}
$$

where

$$
{ }_{k+p} F_{q}=\sum_{i=0}^{[n / k]} \frac{\prod_{r=1}^{p}\left(a_{r}\right)^{(i)}}{\prod_{s=1}^{q}\left(b_{s}\right)^{(i)}} \prod_{j=1}^{k}\left(-\frac{n-j+1}{k}\right)^{(i)} \frac{m^{i}}{i ! x^{k i}},
$$

the generalized hypergeometric Appell polynomials.

We note that if $p=0, q=0, k:=m, m:=(-1)^{k} h k^{k}$ the generalized hypergeometric Appell polynomials $A_{n}^{(k)}(m, x)$ become the Gould-Hopper polynomials $g_{n}^{m}(x, h)$ and if $p=0, q=0$, $m=-2, k=2$ they become the Hermite polynomials $H_{n}(x)$ mentioned above.

The main result of this article is the following basic statement.

Theorem 1. The generalized hypergeometric Appell polynomials $A_{n}^{(k)}(m, x)$ defined by definition 1 are the Appell type ones.

\section{BASIC DEFINITIONS AND NOTATION}

In addition to the rising factorial we use the falling factorial, defined by $(x)_{0}=1$ and $(x)_{n}=x(x-1)(x-2) \cdots(x-n+1)$ for $n>0$. In these notations, the following relations hold (see [1])

$$
(x)_{n}=(-1)^{n}(-x)^{(n)},
$$


and the Gauss product of indexes formula (see [14]) will be written as follows

$$
(-\lambda)^{(m n)}=m^{m n} \prod_{j=1}^{m}\left(-\frac{\lambda-j+1}{m}\right)^{(n)}, \quad n \in \mathbb{N}_{0} .
$$

We note that in the case, when either $a$ or $b$ is a non-positive integer, the generalized hypergeometric function reduces to a polynomial

$$
{ }_{p} F_{q}\left[\begin{array}{c}
-m, a_{2}, \ldots, a_{p} \\
b_{1}, b_{2}, \ldots, b_{q}
\end{array} \mid z\right]=\sum_{n=0}^{\infty}(-1)^{n}\left(\begin{array}{c}
m \\
n
\end{array}\right) \frac{\prod_{j=2}^{p} a_{j}^{(n)}}{\prod_{s=1}^{q} b_{s}^{(n)}} z^{n} .
$$

As far as we deal with the differentiation, the differentiation formula with respect to $z$ would be useful: $\frac{d}{d x} p F_{q}\left[\begin{array}{c}a_{1}, a_{2}, \ldots, a_{p} \\ b_{1}, b_{2}, \ldots, b_{q}\end{array} \mid z\right]=\frac{\prod_{j=1}^{p} a_{j}}{\prod_{s=1}^{q} b_{s}} p F_{q}\left[\begin{array}{c}a_{1}+1, a_{2}+1, \ldots, a_{p}+1 \\ b_{1}+1, b_{2}+1, \ldots, b_{q}+1\end{array} \mid z\right]$ [12].

\section{BASIC PROPERTIES OF THE GENERALIZED HYPERGEOMETRIC APPELL POLYNOMIALS}

\subsection{Being of Appell type}

Proof of Theorem 1. To prove the generalized hypergeometric Appell polynomials $A_{n}^{(k)}(m, x)$ are the Appell-type polynomials, it is sufficient to show that there exists a formal power series $A(t)$ such that the following relation holds

$$
A(t) e^{x t}=\sum_{n=0}^{\infty} A_{n}^{(k)}(m, x) \frac{t^{n}}{n !} .
$$

We set $(\gamma)^{i}=\left(\prod_{r=1}^{p}\left(a_{r}\right)^{(i)}\right) /\left(\prod_{s=1}^{q}\left(b_{s}\right)^{(i)}\right)$. Then from definition 2 and relations (5) and (6) it follows that

$$
A_{n}^{(k)}(m, x)=x_{p+k}^{n} F_{q}\left[\begin{array}{c|c}
a_{1}, a_{2}, \ldots, a_{p}, \Delta(k,-n) \\
b_{1}, b_{2}, \ldots, b_{q}
\end{array} \mid \frac{m}{x^{k}}\right]=x^{n} \sum_{i=0}^{[n / k]} \frac{(\gamma)^{i}(-1)^{k i}(n)_{k i}}{k^{k i}} \frac{m^{i}}{i ! x^{k i}} .
$$

We choose

$$
A(t)={ }_{p} F_{q}\left[\begin{array}{c}
a_{1}, a_{2}, \ldots, a_{p} \\
b_{1}, b_{2}, \ldots, b_{q}
\end{array} \mid(-1)^{k} m \frac{t^{k}}{k^{k}}\right] .
$$

Using the expansion of $e^{x t}$ into the power series and changing the product of the series by the double series, we transform the generating function as follows

$$
A(t) e^{x t}=\left(\sum_{n=0}^{\infty}(\gamma)^{n} \frac{\left((-1)^{k} m \frac{t^{k}}{k^{k}}\right)^{n}}{n !}\right)\left(\sum_{s=0}^{\infty} \frac{(x t)^{s}}{s !}\right)=\sum_{n=0}^{\infty}\left(\sum_{s=0}^{\infty}(\gamma)^{n}(-1)^{k n} \frac{m^{n} x^{s}}{k^{k n}} \frac{t^{s+k n}}{s ! n !}\right)
$$

Using the infinite sums interchange formula ([5]) $\sum_{n=0}^{\infty} \sum_{m=0}^{\infty} a_{n, m}=\sum_{p=0}^{\infty} \sum_{q=0}^{p} a_{p-q, q}$ and taking into account the multiplicity of $i$, we have

$$
\sum_{n=0}^{\infty} \sum_{s=0}^{\infty} a_{s, n}=\sum_{n=0}^{\infty} \sum_{i=0}^{[n / k]} a_{n-k i, i}
$$


then

$$
\begin{aligned}
& \sum_{n=0}^{\infty}\left(\sum_{s=0}^{\infty}(\gamma)^{n} \frac{m^{n}(-1)^{k n} x^{s}}{k^{k n}} \frac{t^{s+k n}}{s ! n !}\right)=\sum_{n=0}^{\infty}\left(\sum_{i=0}^{[n / k]} \frac{m^{i}(-1)^{k i}(\gamma)^{i}}{k^{k i}} \frac{x^{n-k i} t^{n}}{(n-k i) ! i !}\right) \\
& =\sum_{n=0}^{\infty} x^{n}\left(\sum_{i=0}^{[n / k]} \frac{n !}{(n-k i) ! i !} \frac{m^{i}(-1)^{k i}(\gamma)^{i}}{k^{k i}} \frac{1}{x^{k i}}\right) \frac{t^{n}}{n !}=\sum_{n=0}^{\infty} x^{n}\left(\sum_{i=0}^{[n / k]}(\gamma)^{i} \frac{m^{i}(-1)^{k i}(n)_{k i}}{k^{k i}} \frac{\frac{1}{x^{k i}}}{i !}\right) \frac{t^{n}}{n !} .
\end{aligned}
$$

The inner sum is precisely equal to the generalized hypergeometric function in the form of (3) and, therefore, the relation (4) holds. This means that the generating function admit the needed representation (3).

It should be noted that there is another way to prove Theorem 1 , which is to replace $x t$ by $t$ and $m / x^{k}$ by $x$ in [15, Problem 26, p.173].

As a consequence of Theorem1, we derive a new identity for the generalized hypergeometric function.

Corollary 1. The following identity holds

$$
\begin{aligned}
& n x_{p+k}^{n-1} F_{q}\left[\begin{array}{c|c}
a_{1}, a_{2}, \ldots, a_{p}, \Delta(k,-n+1) \\
b_{1}, b_{2}, \ldots, b_{q}
\end{array} \mid \frac{m}{x^{k}}\right]=n x_{p+k}^{n-1} F_{q}\left[\begin{array}{c}
a_{1}, a_{2}, \ldots, a_{p}, \Delta(k,-n) \mid \frac{m}{x^{k}} \\
b_{1}, b_{2}, \ldots, b_{q}
\end{array}\right] \\
& -k m(\gamma)^{1} \Delta_{1}(k,-n) x_{p+k}^{n-k-1} F_{q}\left[\begin{array}{c|c}
a_{1}+1, a_{2}+1, \ldots, a_{p}+1, \Delta(k,-n+k) \\
b_{1}+1, b_{2}+1, \ldots, b_{q}+1
\end{array} \mid \frac{m}{x^{k}}\right],
\end{aligned}
$$

where $\Delta_{1}(k,-n)$ denotes the product $\left(-\frac{n}{k}\right) \cdot\left(-\frac{n-1}{k}\right) \ldots\left(-\frac{n-k+1}{k}\right)$.

Proof. The generalized hypergeometric Appell polynomials are the Appell-type ones, hence, the identity $\frac{d}{d x}\left\{A_{n}^{(k)}(m, x)\right\}=n A_{n-1}^{(k)}(m, x)$ fulfils.

Representing the polynomials $A_{n-1}^{(k)}(m, x)$ in the terms of the generalized hypergeometric function according to Definition 1, we immediately obtain the left side of the corollary equality.

To obtain its right side we differentiate the hypergeometric representation of the polynomials $A_{n}^{(k)}(m, x)$ under the Leibnitz rule:

$$
\begin{aligned}
\frac{d}{d x}\left\{x _ { p + k } ^ { n } F _ { q } \left[\begin{array}{c}
\left.\left.a_{1}, a_{2}, \ldots, a_{p}, \Delta(k,-n) \mid \frac{m}{x^{k}}\right]\right\}= \\
b_{1}, b_{2}, \ldots, b_{q}
\end{array}\right.\right. & n x_{p+k}^{n-1} F_{q}\left[\begin{array}{c}
\left.a_{1}, a_{2}, \ldots, a_{p}, \Delta(k,-n) \mid \frac{m}{x^{k}}\right] \\
b_{1}, b_{2}, \ldots, b_{q}
\end{array}\right. \\
& +x^{n} \frac{d}{d x}\left\{p+k F_{q}\left[\begin{array}{c}
a_{1}, a_{2}, \ldots, a_{p}, \Delta(k,-n) \mid \frac{m}{x^{k}} \\
b_{1}, b_{2}, \ldots, b_{q}
\end{array}\right]\right\} .
\end{aligned}
$$

Performing the derivative of the hypergeometric function, we obtain

$$
\begin{aligned}
x^{n} \frac{d}{d x}\left\{p+k F_{q}\left[\begin{array}{c}
a_{1}, a_{2}, \ldots, a_{p}, \Delta(k,-n) \\
b_{1}, b_{2}, \ldots, b_{q}
\end{array} \mid \frac{m}{x^{k}}\right]\right\}=x^{n}\left(\frac{(-1)^{k}(n)_{k}}{k^{k}} \frac{a_{1} \cdots a_{p}}{b_{1} \cdots b_{q}} \frac{m(-k)}{1 ! x^{k+1}}\right. \\
+\frac{(-1)^{2 k}(n)_{2 k}}{k^{2 k}} \frac{a_{1}\left(a_{1}+1\right) \cdots a_{p}\left(a_{p}+1\right)}{b_{1}\left(b_{1}+1\right) \cdots b_{q}\left(b_{q}+1\right)} \frac{m^{2}(-2 k)}{2 ! x^{2 k+1}} \\
\left.+\frac{(-1)^{3 k}(n)_{3 k}}{k^{3 k}} \frac{a_{1}\left(a_{1}+1\right)\left(a_{1}+2\right) \cdots a_{p}\left(a_{p}+1\right)\left(a_{p}+2\right)}{b_{1}\left(b_{1}+1\right)\left(b_{1}+2\right) \cdots b_{q}\left(b_{q}+1\right)\left(b_{q}+2\right)} \frac{m^{3}(-3 k)}{3 ! x^{3 k+1}}+\cdots\right)
\end{aligned}
$$




$$
\begin{gathered}
=x^{n-k-1} m k \frac{(-1)^{k+1}(n)_{k}}{k^{k}} \frac{a_{1} \cdots a_{p}}{b_{1} \cdots b_{q}}\left(1+\frac{(-1)^{k}(n-k)_{k}}{k^{k}} \frac{\left(a_{1}+1\right) \cdots\left(a_{p}+1\right)}{\left(b_{1}+1\right) \cdots\left(b_{q}+1\right)} \frac{m \cdot 2}{2 ! x^{k}}\right. \\
\left.+\frac{(-1)^{2 k}(n-k)_{2 k}}{k^{2 k}} \frac{\left(a_{1}+1\right)\left(a_{1}+2\right) \cdots\left(a_{p}+1\right)\left(a_{p}+2\right)}{\left(b_{1}+1\right)\left(b_{1}+2\right) \cdots\left(b_{q}+1\right)\left(b_{q}+2\right)} \frac{m^{2} \cdot 3}{3 ! x^{2 k}}+\cdots\right) \\
=-k m(\gamma)^{1} \Delta_{1}(k,-n) x_{p+k}^{n-k-1} F_{q}\left[\begin{array}{c}
a_{1}+1, a_{2}+1, \ldots, a_{p}+1, \Delta(k,-n+k) \\
b_{1}+1, b_{2}+1, \ldots, b_{q}+1
\end{array} \mid \frac{m}{x^{k}}\right],
\end{gathered}
$$

that ends the proof.

Since an arbitrary polynomial of one variable $P_{n}(x) \in \mathbb{C}[x]$ always permits the formal series representation

$$
P_{n}(x)=\sum_{i=0}^{n} \alpha_{i} x^{i}
$$

we are interested in finding those representation for the generalized hypergeometric Appell polynomials.

Corollary 2. The generalized hypergeometric Appell polynomials $A_{n}^{(k)}(m, x)$ possess

(i) the standard basis $\left\{x^{i}\right\}_{i=0}^{n}$ representation

$$
A_{n}^{(k)}(m, x)=\sum_{i=0}^{[n / k]} \frac{n !(-1)^{k i}(\gamma)^{i} m^{i}}{i ! k^{k i}(n-k i) !} x^{n-k i},
$$

(ii) the differential operator formal power series representation

$$
A_{n}^{(k)}(m, x)=\left(\sum_{i=0}^{[n / k]} \frac{(-1)^{k i}(\gamma)^{i} m^{i}}{i ! k^{k i}} D^{k i}\right) x^{n}
$$

Proof. (i) We use an approach from [6], which is based on the idea of the connection problem.

Given the two polynomial families of Appell type $\left\{P_{n}(x)\right\}$ and $\left\{Q_{n}(x)\right\}$ with generating functions $A_{1}(t)$ and $A_{2}(t)$ respectively, the solution of its connection problem could be written as follows

$$
Q_{n}(x)=\sum_{m=0}^{n} \frac{n !}{m !} \alpha_{n-m} P_{m}(x),
$$

where $\frac{A_{2}(t)}{A_{1}(t)}=\sum_{k=0}^{\infty} \alpha_{k} t^{k}$.

We are searching for the unknown coefficients $\alpha_{k}$ to decompose the polynomials

$$
Q_{n}(x)=x^{n}, A_{2}(t)=1
$$

upon the polynomials $A_{n}^{(k)}(m, x)$ defined by (3) with generating function $A_{1}(t)$ defined by (7). Deriving the ratio of generating functions $A_{2}(t)$ and $A_{1}(t)$ we have

$$
\frac{A_{2}(t)}{A_{1}(t)}=\sum_{r=0}^{\infty} \frac{(-1)^{k r} m^{r}}{k^{k r} r !}(\gamma)^{r} t^{k r}=\sum_{r=0}^{\infty} \alpha_{r k} t^{r k},
$$


and, constructing the corresponding coefficients $\alpha_{n-m}$, we obtain the needed representation.

(ii) An arbitrary Appell-type polynomial $P_{n}(x)$ could be also written in the symmetric form

$$
P_{n}(x)=\sum_{i=0}^{n}\left(\begin{array}{c}
n \\
i
\end{array}\right) c_{i} x^{n-i}
$$

According to [11], the latter expression is equivalent to the following differential operator representation

$$
P_{n}(x)=\left(\sum_{i=0}^{n} \frac{c_{i}}{i !} D^{i}\right) x^{n}
$$

where $D:=d / d x$ is an ordinary differentiation with respect to $x$, consequently,

$$
A_{n}^{(k)}(m, x)=\sum_{i=0}^{[n / k]}\left(\begin{array}{c}
n \\
k i
\end{array}\right) c_{i} x^{n-k i}=\sum_{i=0}^{[n / k]}\left(\begin{array}{c}
n \\
k i
\end{array}\right) \frac{(-1)^{k i}(\gamma)^{i} m^{i}(k i) !}{i ! k^{k i}} x^{n-k i},
$$

we deduce a differential operator formal power series representation of the generalized hypergeometric Appell polynomials of the form (9).

Remark. Comparing the power series (1) and operational formula (9) of the generalized hypergeometric Appell polynomials to the corresponding ones of the Gould-Hopper polynomials

$$
A(t)=e^{h t^{m}}, \quad g_{n}^{m}(x, h)=\left(e^{h D^{m}}\right) x^{n},
$$

it is easy to see that the latter have more compact forms.

Symmetry. Substituting the negative value of argument into the formula (8)

$$
A_{n}^{(k)}(m,-x)=\sum_{i=0}^{[n / k]}(-1)^{n-k i} \frac{n !(-1)^{k i}(\gamma)^{i} m^{i}}{i ! k^{k i}(n-k i) !} x^{n-k i}
$$

we conclude that, in the case of even $k$, the generalized hypergeometric Appell polynomials are the even ones themselves while $n$ is an even number, and they are the odd ones themselves while $n$ is an odd number:

$$
A_{2 n}^{(2 k)}(m,-x)=A_{2 n}^{(2 k)}(m, x), \quad A_{2 n+1}^{(2 k)}(m,-x)=-A_{2 n+1}^{(2 k)}(m, x) .
$$

Otherwise, for any odd $k$ in the case of odd $n$, the summands standing on the even places change their signs into the opposite ones, and the same do the summands standing on the odd places in the case of even $n$.

\subsection{Adition and multiplication formulas and other properties}

Here we shall prove the following result.

Theorem 2. The following formulas hold for the generalized hypergeometric Appell polynomials

(i) addition formula

$$
A_{n}^{(k)}(m, x+y)=\sum_{i=0}^{n}\left(\begin{array}{c}
n \\
i
\end{array}\right) y^{n-i} A_{i}^{(k)}(m, x)=\sum_{i=0}^{n}\left(\begin{array}{c}
n \\
i
\end{array}\right) x^{n-i} A_{i}^{(k)}(m, y),
$$


(ii) multiplication formula

$$
A_{n}^{(k)}(m, M x)=\sum_{i=0}^{n}\left(\begin{array}{c}
n \\
i
\end{array}\right)(M-1)^{n-i} x^{n-i} A_{i}^{(k)}(m, x)
$$

(iii) indexes interchange formula

$$
\sum_{i=0}^{n}\left(\begin{array}{c}
n \\
i
\end{array}\right) A_{i}^{\left(k_{1}\right)}(m, x) A_{n-i}^{\left(k_{2}\right)}(m, y)=\sum_{i=0}^{n}\left(\begin{array}{c}
n \\
i
\end{array}\right) A_{i}^{\left(k_{2}\right)}(m, x) A_{n-i}^{\left(k_{1}\right)}(m, y),
$$

(iv) convolution type identity

$$
\begin{aligned}
\sum_{i=0}^{n}(-1)^{i}\left(\begin{array}{c}
n \\
i
\end{array}\right) & A_{i}^{(k)}(m, x) A_{n-i}^{(k)}(m, x) \\
& =\frac{(-1)^{n} m^{n / k} n !}{k^{n}} \sum_{i=0}^{[n / k]} \frac{a_{1}^{(i)} \ldots a_{p}^{(i)}}{i ! b_{1}^{(i)} \ldots b_{q}^{(i)}} \frac{a_{1}^{(n / k-i)} \ldots a_{p}^{(n / k-i)}}{(n / k-i) ! b_{1}^{(n / k-i)} \ldots b_{q}^{(n / k-i)}} .
\end{aligned}
$$

Proof. The addition and the multiplication formulas hold for all Appell-type polynomial families ([11]), consequently, they hold for the generalized hypergeometric Appell polynomials as well. The indexes interchange formulas could be obtained applying methods proposed in [6] and the convolution type identity is obtained by the direct calculations at $x=0$.

It is worth stressing, that the polynomials $A_{n}^{(k)}(m, M x)$ loose the property of being of Appell-type. Moreover, the generalized hypergeometric polynomials over the polynomials could be defined in the same manner as the generalized hypergeometric Appell polynomials:

$$
A_{n}^{(k)}(m, f(x))=(f(x))_{p+k}^{n} F_{q}\left[\begin{array}{c|c}
a_{1}, a_{2}, \ldots, a_{p}, \Delta(k,-n) & \frac{m}{(f(x))^{k}}
\end{array}\right],
$$

where $f(x)=a_{0} x^{p}+a_{1} x^{p-1}+\cdots+a_{p}, \quad a_{0} \neq 0$, which deliver us the following differentiation rule

$$
\frac{d}{d x} A_{n}^{(k)}(m, f(x))=n f^{\prime}(x) A_{n-1}^{(k)}(m, f(x)) .
$$

In particular, in the case when $p=a_{0}=1$, we obtain the Appell differentiation.

\section{REFERENCES}

[1] Abramowitz M., Stegun I.A. Handbook of mathematical functions with Formulas, Graphs, and Mathematical Tables. Dover Publications, New York, 1972.

[2] Aceto L., Malonek H.R., Tomaz Gr. A unified matrix approach to the representation of Appell polynomials. Integral Transforms Spec. Funct. 2015, 26 (6), 426-441. doi:10.1080/10652469.2015.1013035

[3] Aldo F., Longo E. An algebraic approach to Sheffer polynomial sequences. Integral Transforms Spec. Funct. 2014, 25 (4), 295-311. doi:10.1080/10652469.2013.842234

[4] Appell P. On one class of polynomials. Annales scientifiques de 1'E.N.S. 2e serie 1880, 9, 119-144.

[5] Arfken G.B., Weber H.J., Harris F.E. Mathematical Methods for Physicists: A Comprehensive Guide. Academic Press, 7th edition, 2011. 
[6] Cheikh Y.B., Chaggara H. Connection problems via lowering operators. J. Comput. Appl. Math. 2005, 178 (1-2), 45-61. doi:10.1016/j.cam.2004.02.024

[7] Costabile F.A., Longo E. A determinantal approach to Appell polynomials. J. Comput. Appl. Math. 2010, 234 (5), 528-1542. doi:10.1016/j.cam.2010.02.033

[8] Dominici D. Asymptotic analysis of generalized Hermitepolynomials. Analysis (Berlin) 2008, 28 (2), $239-261$. doi:10.1524/anly.2008.0911

[9] Fasenmyer M.C. Some generalized hypergeometric polynomials. Bull. Amer. Math. Soc. 1947, 8 (53), $806-812$.

[10] Gould H.W., Hopper A.T. Operational formulas connected with two generalizations of Hermite Polynomials. Duke Math. J. 1962, 29, 51-63.

[11] Hazewinkel M. Appell polynomials: Encyclopedia of Mathematics. Kluwer Academic Publishers, 2001.

[12] Slater L.J. Generalized hypergeometric functions. Cambridge University Press, Cambridge, UK, 1966.

[13] Srivastava R. Some generalizations of Pochhammer's symbol and their associated families of hypergeometric functions and hypergeometric polynomials. Appl. Math. Inf. Sci. 2013, 7 (6), 2195-2206.

[14] Srivastava H.M., Karlsson W. Multiple Gaussian Hypergeometric. Series: Ellis Horwood Series in Mathematics and Its Applications. Ellis Horwood, Ltd. 1994.

[15] Srivastava H.M., Manocha H.L. A treatise on generating functions. Halsted Press, New York, 1984.

[16] Yang Y., Micek C. Generalized Pascal functional matrix and its applications. Linear Algebra Appl., 2007, 423, 230-245. doi:10.1016/j.laa.2006.12.014

Received 30.05.2019

Revised 21.12.2019

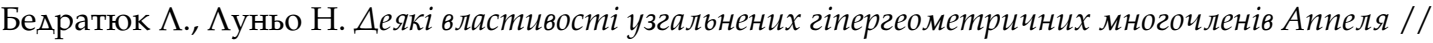
Карпатські матем. публ. - 2020. - Т.12, №1. - С. 129-137.

У цій статті ми представляємо нове сімейство многочленів типу Аппеля $\left\{A_{n}^{(k)}(m, x)\right\}$, $n, m \in \mathbb{N}_{0}, k \in \mathbb{N}$, кожен представник якого визначений над полем дійсних чисел і може бути представлений через узагальнену гіпергеометричну функцію

$$
{ }_{p} F_{q}\left[\begin{array}{c}
a_{1}, a_{2}, \ldots, a_{p} \\
b_{1}, b_{2}, \ldots, b_{q}
\end{array} \mid z\right]=\sum_{k=0}^{\infty} \frac{a_{1}^{(k)} a_{2}^{(k)} \ldots a_{p}^{(k)}}{b_{1}^{(k)} b_{2}^{(k)} \ldots b_{q}^{(k)}} \frac{z^{k}}{k !},
$$

де через $x^{(n)}$ позначено символ Похгаммера (зростаючий факторіал), який визначають за формулою $x^{(n)}=x(x+1)(x+2) \cdots(x+n-1)$ для $n \geq 1$ і $x^{(0)}=1$, у такий спосіб

$$
A_{n}^{(k)}(m, x)=x_{k+p}^{n} F_{q}\left[\begin{array}{c}
\left.a_{1}, a_{2}, \ldots, a_{p},-\frac{n}{k},-\frac{n-1}{k}, \ldots,-\frac{n-k+1}{k} \mid \frac{m}{x^{k}}\right], \\
b_{1}, b_{2}, \ldots, b_{q}
\end{array}\right]
$$

і одночасно многочлени цього сімейства є многочленами типу Аппеля.

Аля многочленів цього сімейства вперше знайдено породжуючу функцію і доведено, що вони є многочленами типу Аппеля. Знайдено розклад представників цього сімейства за стандартним базисом в замкнутій формі та у формі ряду диференціального оператора, а також нову тотожність для узагальненої гіпергеометричної функції. Крім цього, для узагальнених гіпергеометричних многочленів Аппеля встановлено формули додавання і множення аргумента та деякі інші.

Ключові слова і фрази: послідовність Аппеля, многочлен Аппеля, узагальнений гіпергеометричний многочлен, узагальнена гіпергеометрична функція. 